\title{
Introduction : Le Grand Est, lecture géographique d'un espace en émergence
}

Introduction: Great East, geographical reading of an emergent space

Einleitung: Der Grosse Osten, geographisches Lesen von einem auftauchenden

Raum

Christiane Rolland-May

\section{(2) OpenEdition}

Journals

Édition électronique

URL : http://journals.openedition.org/rge/617

DOI : $10.4000 /$ rge.617

ISSN : 2108-6478

Éditeur

Association des géographes de l'Est

Édition imprimée

Date de publication : 1 juin 2004

Pagination : 93-95

ISSN : 0035-3213

Référence électronique

Christiane Rolland-May, «Introduction : Le Grand Est, lecture géographique d'un espace en émergence », Revue Géographique de l'Est [En ligne], vol. 44 / 3-4 | 2004, mis en ligne le 28 mai 2009, consulté le 08 septembre 2020. URL : http://journals.openedition.org/rge/617 ; DOI : https://doi.org/ $10.4000 /$ rge.617

Ce document a été généré automatiquement le 8 septembre 2020

Tous droits réservés 


\title{
Introduction : Le Grand Est, lecture géographique d'un espace en émergence
}

Introduction: Great East, geographical reading of an emergent space

Einleitung: Der Grosse Osten, geographisches Lesen von einem auftauchenden

Raum

\author{
Christiane Rolland-May
}

1 Ce numéro de la RGE se propose de faire une lecture géographique du Grand Est de la France. L'objectif poursuivi est de mettre en évidence les spécificités, la complexité spatiale et systémique, les potentialités en matière d'aménagement et de développement de ce grand espace, mais également de voir s'il est possible d'en dégager une réelle identité géographique et territoriale. La lecture menée sera celle de géographes, soucieux à la fois de recherche fondamentale et d'approches en aménagement. Nous la voulons en "regards croisés", c'est-à-dire que des problématiques volontairement variées seront ouvertes et traitées à travers des thématiques diverses propres au domaine de recherche de chaque auteur. La lecture proposée sera également multi-échelles : elle concernera essentiellement le Grand Est dans son ensemble (articles de H. Nonn, S. de Ruffray, C. Rolland-May et S. Lebahar, L. Bertrand), mais l'étude d'un exemple plus précis et plus localisé apportera un éclairage particulièrement significatif sur un territoire qui, de façon quasi fractale, reproduit à l'échelle locale les problématiques globale du Grand Est (article de G. Cohen, B. Reitel et R. Woessner). Un dernier travail (article de C. Sohn) se placera dans une logique de "système multi-agents » en abordant par un exemple le jeu des acteurs dans la construction d'un territoire.

2 Les auteurs sont néanmoins conscients que, dans le cadre limité de cette publication, l'ambition est nécessairement limitée et qu'ils ne peuvent que proposer une esquisse en petites touches de ce grand espace, qui, malgré son fort potentiel géostratégique à l'échelle européenne, reste encore un « espace en émergence ». 


\section{Une difficile définition : le Grand Est, un « espace géographique flou »}

3 Dans le contexte actuel de recomposition des territoires, force est de constater que le «Grand Est » de la France est une entité spatiale floue, mal identifiée et en définitive relativement peu appropriée par les chercheurs et les acteurs de l'aménagement. Quatre points majeurs méritent attention.

4 En premier lieu, le facteur essentiel de mauvaise lisibilité géographique du Grand Est réside dans le fait que ce grand espace peut être présenté comme un «espace géographique flou », que nous avons défini par ailleurs et depuis fort longtemps comme un espace mal délimité par rapport à son environnement, ou dont les limites sont mal ou incomplètement perçues par l'observateur. Dans notre cas l'imprécision spatiale du Grand Est a des causes multiples et bien connues: manque d'unité géographique, flottement historique lié à la mobilité des frontières qui induit l'absence de mémoire collective et de communauté historique, complexité du cadrage institutionnel, morcellement des réseaux et territoires, etc. La définition du Grand Est qui en résulte est nécessairement plurielle. Quelles sont les composantes spatiales de cette entité ? S'agit-il d'un "grand»Grand Est recouvrant les cinq régions (Champagne-Ardenne, Lorraine, Alsace, Franche-Comté, Bourgogne), ainsi que l'entend la Datar, soit un ensemble de 18 départements, de près de $13 \%$ de la population de la France sur $20 \%$ de sa superficie? S'agit-il d'une entité plus réduite, composée des trois régions de l'est de notre pays (Lorraine, Alsace, Franche-Comté)? S'agit-il enfin du «noyau dur» représenté par l'Alsace et la Lorraine? La diversité des réponses apportées dans les travaux scientifiques et dans la constitution d'organismes, institutions et autres structures interrégionales qui se recommandent toutes du concept de "Grand Est » éclaire de façon significative ce flou de la configuration spatiale de notre espace d'étude.

5 En étroite corrélation avec ce premier constat, soulignons à présent la complexité institutionnelle du territoire. La prégnance et la complexité de l'entrelacs des limites administratives contrarient la vision d'ensemble, enferment les projets à l'intérieur des entités administratives et bloquent les actions innovantes. On insistera tout particulièrement sur les limites régionales, véritables lignes de discontinuités spatiales, si bien que les recompositions territoriales qui cherchent et arrivent à dépasser ces barrières et à s'inscrire dans une logique suprarégionale sont rares. Sans être déterminant, le cadrage institutionnel de l'espace représente ainsi un facteur de nonou de mauvaises visibilité et lisibilité du territoire concerné.

6 En troisième lieu, rappelons que la perception globale du Grand Est est également troublée par la complexité de l'organisation urbaine et la configuration des aires polarisées qui en résulte. Ce polycentrisme de métropoles plus concurrentes que complémentaires s'enracine dans les spécificités géo-socio-historiques de l'aire culturelle rhénane, ainsi que les travaux d'E. Juillard et de l'école géographique de Strasbourg l'ont magistralement démontré. Il en résulte un morcellement de l'espace organisé du Grand Est, localement bien structuré en réseaux urbains hiérarchisés et fonctionnels et en territoires métropolisés nets, mais globalement segmenté du fait de la faible inter-articulation de ces réseaux, "troué " par des espaces peu irrigués par la métropolisation qui apparaissent comme des «zones blanches» sur la carte des 
structures urbaines. Le Grand Est ne perpétue-t-il pas de ce fait la réalité de l'expression de "régionsde l'Est", le pluriel employé à dessein par E. Juillard ne représente-t-il pas une des caractéristiques invariantes de notre espace d'étude?

7 En complément aux réponses apportées à ces questionnements, force est de constater en dernier lieu que cet espace est fortement dépendant de structures, d'organisations spatiales et de dynamiques exogènes, qui masquent l'identité globale du Grand Est et en font plus un espace de marge à forts morcellement interne et effritement périphérique qu'un ensemble véritablement cohérent. Les dynamiques externes, qu'elles soient d'échelle nationale (attraction de Paris, dynamique rhodanienne), transnationale (dynamiques transfrontalières), continentale (dynamiques des flux drainés par l'isthme rhénan), sont génératrices de contacts, d'échanges et de processus d'innovations dans les secteurs les plus ouverts et les mieux interconnectés du Grand Est. En revanche, les espaces à faible organisation urbaine (Meuse et bordure ouest du Grand Est, plateaux de Haute Saône) sont soumis à des processus centrifuges d'aspiration de leurs ressources humaines et forces vives, ou ne profitent que façon médiocre des flux qui les traversent (« effet tunnel »). L'article présenté par $\mathbf{S}$. de Ruffray est significatif à ce point de vue. On y souligne que la position de marge que connaît le Grand Est apparaît à la fois comme un facteur à la fois défavorable pour un développement global de cet espace et comme un générateur d'opportunités, à condition cependant que les villes du Grand Est, estimées trop peu puissantes, se structurent en réseaux et que se renforcent des agglomérations transfrontalières. Dans ce cas, et dans ce cas seulement, souligne cet auteur, le Grand Est sera à même de valoriser sa position d'interface et de relais actif entre les espaces forts voisins, nationaux ou internationaux.

\section{II. Élements de lecture globale du Grand Est}

8 Dans cette optique, soulignons l'intérêt de l'article de H. Nonn. Partant des travaux récents de la Datar sur l'aménagement de la France pour 2020 et s'appuyant sur sa connaissance approfondie du Grand Est affinée encore par sa longue collaboration avec cette institution, l'auteur travaille à promouvoir l'idée d'un Grand Est à organisation urbaine cohérente fondée sur un "polycentrisme maillé » dont il explicite le principe. Il souligne en second lieu la nécessité d'une logique de constructions territoriales adaptées au contexte géo-socio-économique et géopolitique actuel, l'Europe élargie exigeant à plus ou moins long terme que soient définies ou redéfinies de nouvelles échelles de cohérence des espaces européens. Le Grand Est apparaît fort justement comme une aire de pertinence en phase avec la nouvelle donne européenne. Ainsi cadré, notre espace d'étude relève d'une approche scientifiquement et stratégiquement innovante: celle d'un territoire français construit à l'échelle européenne, la construction devant associer l'ensemble des acteurs institutionnels, économiques, sociaux, quelle que soit leur propre échelle d'intervention et d'action.

Pour sa part, L. Bertrand s'attache à développer l'idée que l'intégration transfrontalière représente un facteur de mise en cohérence du Grand Est, car elle permettra à cet espace d'exercer pleinement sa vocation européenne, vocation autorisée par la large ouverture frontalière du Grand Est et les relations privilégiées anciennes et diversifiées entretenues avec les pays voisins. Cette lecture est nécessairement trans-scalaire et se décline en projets d'intégration locales (agglomérations transfrontalières), régionale (construction d'euro-régions) et globale 
(rôle du Grand Est comme médiateur et interface dans la construction européenne). La dimension transfrontalière du Grand Est peut ainsi apporter des éléments de réponse à l'émergence d'une identité globale de l'espace étudié.

C. Rolland-May et S. Lebahar proposent une autre grille de lecture du Grand Est. S'attachant à l'analyse des sites Internet développés par les centres urbains et les établissements publics de coopération intercommunale, les auteurs s'intéressent aux rapports entre Géographie et organisation de la «Toile » (le réseau formé par les sites) et développent une méthodologie d'approche spécifique. Deux points essentiels sont mis en exergue. En premier lieu les auteurs soulignent que la donne géographique est retranscrite dans l'organisation de la Toile, mais que cette retranscription d'une réalité spatiale en une réalité « immatérielle » se traduit par un gain de complexité qu'elles s'efforcent d'identifier et de caractériser. En second lieu elles soulignent que l'organisation de la Toile elle-même est de nature géographique et en présentent une visualisation cartographique significative.

\section{Contribution de l'échelle locale à l'approche du Grand Est}

11 L'exemple du Haut-Rhin, de son positionnement et de ses stratégies de développement développé par G. Cohen, B. Reitel et R. Woessner réalise un « effet de zoom » sur ce département, territoire particulièrement stratégique du fait de son positionnement géo-économique transfrontalier. Les auteurs en soulignent les caractéristiques majeures: dépendance extérieure en matière de développement industriel, forte urbanisation et absence de véritables métropoles, mais aussi mutation récente des stratégies volontaristes de développement, accent mis sur la mobilisation des énergies infra-territoriales, en particulier sur les dynamiques participatives des acteurs et sur les processus d'innovation. Passant du «local » au «global», ne peut-on considérer ce territoire d'étude comme représentatif de l'ensemble du Grand Est et, le cas échéant, lire les «nouvelles perspectives de développement du Haut-Rhin » présentées par les trois auteurs comme un ensemble de propositions généralisables à notre grand espace ? Sans être en mesure d'apporter une réponse ferme à la portée générale des propos et propositions énoncés, soulignons que ce questionnement interpelle le chercheur et appellera sans doute une réflexion approfondie et d'autres travaux.

\section{Le Grand Est, une réalité (incomplète) façonnée par les acteurs}

12 En dernier ressort il est notable que le concept de Grand Est est progressivement approprié par les acteurs du territoire. Au niveau global, même s'ils le définissent de façon imparfaite par rapport à nos critères géographiques, ils utilisent le concept de Grand Est comme une échelle de pertinence pour atteindre une masse critique suffisante et exercer un lobbying efficace (Association des Régions françaises du Grand Est, Réunions des présidents des CESR), justifier d'investissements technologiques lourds (Cancéropole du Grand Est), mutualiser les ressources en "matière grise " (Délégation «Grand Est » du Club de Nanotechnologie), réaliser et organiser au mieux le développement de leurs actions (Réseau des secrétaires généraux et responsables de 
formation). Ces acteurs institutionnels sont timidement relayés par les professionnels, qui trouvent à cette échelle une dimension propice à la mutualisation des moyens et des actions.

Force est de reconnaître cependant qu'au niveau local, la préoccupation de mettre les actions territoriales en cohérence avec l'échelle du Grand Est apparaît ténue, voire inexistante et que les stratégies de développement de territoires n'expriment que peu, voire pas du tout, un souci d'arrimage à une entité en définitive mal perçue, comme nous l'avons vu plus haut. A titre d'illustration, il est significatif de constater que les problématiques transfrontalières ne s'expriment que très rarement à cette échelle, chaque territoire infra-régional ou régional voulant assurer à son échelle et à son profit l'arrimage à des dynamiques exogènes.

Dans cette optique l'article de C. Sohn est significatif d'un exemple de recomposition territoriale d'envergure, celle de la région strasbourgeoise et du SCOTERS, et du déroulement d'une démarche explicitement inscrite dans une stratégie participative et mobilisatrice des acteurs locaux, mais où la vision du Grand Est est singulièrement absente. Quelle est la signification de ce contre-exemple? S'agit-il du constat que le concept de Grand Est reste en définitive relatif à une échelle spatiale privilégiée et qu'il se dissout dans les échelles d'observation et d'action plus grandes et plus petites ? Peuton simplement énoncer l'idée que la réalité de cette entité spatiale et organisationnelle est encore en " en devenir » et que le Grand Est est un " espace en émergence »?

Même si, malgré la diversité des approches thématiques et méthodologiques menées, les travaux présentés n'apportent pas de réponse à ces questionnements, il n'en reste pas moins qu'ils ont le mérite d'ouvrir le débat scientifique sur ce grand espace et d'interroger sur sa réalité et son devenir.

\section{AUTEUR}

\section{CHRISTIANE ROLLAND-MAY}

ULP Strasbourg 1, Faculté de Géographie et d'Aménagement, 3 rue de l'Argonne, 67000 Strasbourg 